\title{
Characterisation of methicillin resistant Staphylococcus aureus clinical isolates from animals in New Zealand, 2012-2013, and subclinical colonisation in dogs and cats in Auckland
}

\author{
A Karkaba ${ }^{* \dagger}, \mathbf{J}$ Benschop $^{\dagger}, \mathrm{KE} \mathrm{Hill}^{\ddagger}$ and A Grinberg ${ }^{*}$ \\ * Institute of Veterinary, Animal and Biomedical Sciences, Infectious Diseases Group, Massey University, Private Bag 11222, Palmerston \\ North 4442, New Zealand. \\ $\dagger$ Institute of Veterinary, Animal and Biomedical Sciences, mEpiLab, Massey University, Private Bag 11222, Palmerston North 4442, New \\ Zealand. \\ $\ddagger$ Current address: School of Veterinary Science, The University of Queensland, Gatton Campus Qld 4343, Australia. \\ \Author for correspondence. Email: A.grinberg@massey.ac.nz
}

Supplementary Table 1. Summary of the DNA microarray and spa results of eight methicillin resistant Staphylococcus aureus clinical isolates.

\begin{tabular}{|c|c|c|c|c|c|c|}
\hline Isolate & 1 & $2 \& 3$ & 4 & $5 \& 5.1$ & 6 & $\mathrm{a}$ \\
\hline \multicolumn{7}{|l|}{ TYPING SUMMARY } \\
\hline strain & $\begin{array}{l}\text { CC5-MRSA-IV, } \\
\text { AK3, or } \\
\text { 'Paediatric } \\
\text { clone' }\end{array}$ & $\begin{array}{l}\text { ST5/ST225- } \\
\text { MRSA-II, } \\
\text { New York- } \\
\text { Japan Clone }\end{array}$ & $\begin{array}{l}\text { CC30-MRSA- } \\
\text { IV [PVL+], } \\
\text { Southwest } \\
\text { Pacific Clone }\end{array}$ & $\begin{array}{l}\text { CC22-MRSA-IV } \\
\text { [fnbB-,sec/l+], } \\
\text { UK-EMRSA- } \\
\text { 15/Barnim } \\
\text { EMRSA }\end{array}$ & $\begin{array}{l}\text { CC8- } \\
\text { MRSA-IV, } \\
\text { USA500 }\end{array}$ & $\begin{array}{l}\text { CC1-MSSA } \\
\text { [lukF- } \\
\text { P83/lukM+] }\end{array}$ \\
\hline strain synonyms & $\begin{array}{l}\text { WA MRSA- } \\
\text { 03/25/50/71/74/ } \\
82 / 105 / 111, \\
\text { USA800, } \\
\text { Spanish PFGE } \\
\text { type E7/8, } \\
\text { Marseille CF } \\
\text { clone }\end{array}$ & $\begin{array}{l}\text { USA100, } \\
\text { Canadian } \\
\text { MRSA-2, Irish } \\
\text { AR7.3/AR7.4, } \\
\text { Finland E1, } \\
\text { JH1/JH9 }\end{array}$ & $\begin{array}{l}\text { USA 1100, } \\
\text { West } \\
\text { Samoan } \\
\text { Phage } \\
\text { Pattern } \\
\text { (WSPP) } \\
\text { Clone }\end{array}$ & $\begin{array}{l}\text { Irish AR06, } \\
\text { Canadian } \\
\text { MRSA-8, } \\
\text { Spanish PFGE } \\
\text { type E13, } \\
\text { "ST22-A" clade } \\
\text { of UK-ERMSA- } \\
15\end{array}$ & $\begin{array}{l}\text { WA MRSA- } \\
20 / 58\end{array}$ & \\
\hline MLST clonal complex affiliation & $\mathrm{CC5}$ & $\mathrm{CC} 5$ & CC30 & CC22 & CC8 & $\mathrm{CC} 1$ \\
\hline $\begin{array}{l}\text { sequence types associated with this } \\
\text { strain }\end{array}$ & $\begin{array}{l}\text { ST5 (based on } \\
\text { whole genome } \\
\text { sequencing) }\end{array}$ & $\begin{array}{l}\text { ST5 (based on } \\
\text { whole genome } \\
\text { sequencing) }\end{array}$ & ST30 & ST22 & ST8 & \\
\hline spa type associated with this strain & t045 & t002 & t019 & t032 & t064 & t144 \\
\hline $\begin{array}{l}\text { assignment score for CC } \\
\text { identification }\end{array}$ & $96.83 \%$ & $97.66 \%$ & $97.52 \%$ & $96.97 \%$ & $94.21 \%$ & $95.04 \%$ \\
\hline MRSA (mecA) & positive & positive & positive & positive & positive & negative \\
\hline MRSA (mecC) & negative & negative & negative & negative & negative & negative \\
\hline PVL & negative & negative & positive & negative & negative & negative \\
\hline \multicolumn{7}{|l|}{ SPECIES MARKER } \\
\hline Target Gene & Result & Result & Result & Result & Result & Result \\
\hline rrnD1 (S. aureus) & positive & positive & positive & positive & positive & positive \\
\hline gapA & positive & positive & positive & positive & positive & positive \\
\hline katA & positive & positive & positive & positive & positive & positive \\
\hline $\operatorname{coA}$ & positive & positive & positive & positive & positive & positive \\
\hline nuc1 & positive & positive & positive & positive & positive & positive \\
\hline spa & positive & positive & positive & positive & positive & positive \\
\hline sbi & positive & positive & positive & positive & positive & positive \\
\hline \multicolumn{7}{|l|}{ REGULATORY GENES } \\
\hline Target Gene & Result & Result & Result & Result & Result & Result \\
\hline sarA & positive & positive & positive & positive & positive & positive \\
\hline saes & positive & positive & positive & positive & positive & positive \\
\hline
\end{tabular}

The content of this supplementary information has not been edited. All risk and liability rest with the authors. 


\section{Supplementary Information}

Karkaba et al.

New Zealand Veterinary Journal

\begin{tabular}{|c|c|c|c|c|c|c|}
\hline vraS & positive & positive & positive & positive & positive & positive \\
\hline agrl (total) & negative & negative & negative & positive & positive & negative \\
\hline agrB-I & negative & negative & negative & positive & positive & negative \\
\hline agrC-1 & negative & negative & negative & ambiguous & positive & negative \\
\hline agrD-I & negative & negative & negative & positive & positive & negative \\
\hline agrll (total) & positive & positive & negative & negative & negative & negative \\
\hline agrB-II & positive & positive & negative & negative & negative & negative \\
\hline agrC-II & positive & positive & negative & negative & negative & negative \\
\hline agrD-II & positive & positive & negative & negative & negative & negative \\
\hline agrlll (total) & negative & negative & positive & negative & negative & positive \\
\hline agrB-III & negative & negative & positive & negative & negative & positive \\
\hline agrC-III & negative & negative & positive & negative & negative & positive \\
\hline agrD-III & negative & negative & positive & negative & negative & positive \\
\hline agrIV (total) & negative & negative & negative & negative & negative & negative \\
\hline agrB-IV & negative & negative & negative & ambiguous & ambiguous & negative \\
\hline agrC-IV & negative & negative & negative & negative & negative & negative \\
\hline hld & positive & positive & positive & positive & positive & positive \\
\hline \multicolumn{7}{|c|}{ METHICILLIN RESISTANCE GENOTYPE AND SCCmec TYPING } \\
\hline Target Gene & Result & Result & Result & Result & Result & Result \\
\hline mecA & positive & positive & positive & positive & positive & negative \\
\hline mecC & negative & negative & negative & negative & negative & negative \\
\hline Isolate & 1 & $2 \& 3$ & 4 & $5 \& 5.1$ & 6 & $\mathrm{a}$ \\
\hline delta_mecR & positive & positive & positive & positive & positive & negative \\
\hline ugpQ & positive & positive & positive & positive & positive & negative \\
\hline ccrA-1 & negative & negative & negative & negative & negative & negative \\
\hline ccrB-1 & negative & negative & negative & negative & negative & negative \\
\hline plsScC (COL) & negative & negative & negative & negative & negative & negative \\
\hline Q9XB68-dcs & positive & positive & positive & positive & positive & negative \\
\hline ccrA-2 & positive & positive & positive & positive & positive & negative \\
\hline ccrB-2 & positive & positive & positive & positive & positive & negative \\
\hline kdpA-SCC & negative & positive & negative & negative & negative & negative \\
\hline kdpB-SCC & negative & positive & negative & negative & negative & negative \\
\hline kdpC-SCC & negative & positive & negative & negative & negative & negative \\
\hline kdpD-SCC & negative & positive & negative & negative & negative & negative \\
\hline kdpE-SCC & negative & positive & negative & negative & negative & negative \\
\hline mecl & negative & positive & negative & negative & negative & negative \\
\hline mecR & negative & positive & negative & negative & negative & negative \\
\hline$x y \mid R$ & negative & positive & negative & negative & negative & negative \\
\hline ccrA-3 & negative & negative & negative & negative & negative & negative \\
\hline ccrB-3 & negative & negative & negative & negative & negative & negative \\
\hline merA & negative & negative & negative & negative & negative & negative \\
\hline merB & negative & negative & negative & negative & negative & negative \\
\hline ccrAA (MRSAZH47)_probe 1 & negative & negative & negative & negative & negative & negative \\
\hline ccrAA (MRSAZH47)_probe 2 & negative & negative & negative & negative & negative & negative \\
\hline ccrC (85-2082) & negative & negative & negative & negative & negative & negative \\
\hline ccrA-4 & negative & negative & negative & negative & negative & negative \\
\hline ccrB-4 & negative & negative & negative & negative & negative & negative \\
\hline \multicolumn{7}{|c|}{ RESISTANCE GENOTYPE : PENICILLINASE } \\
\hline Target Gene & Result & Result & Result & Result & Result & Result \\
\hline blaz & positive & positive & positive & positive & positive & positive \\
\hline blaZ-SCCmec XI & negative & negative & negative & negative & negative & negative \\
\hline blal & positive & positive & positive & positive & positive & positive \\
\hline blaR & positive & positive & positive & positive & positive & positive \\
\hline \multicolumn{7}{|c|}{ RESISTANCE GENOTYPE : MLS-ANTIBIOTICS } \\
\hline Target Gene & Result & Result & Result & Result & Result & Result \\
\hline $\operatorname{erm}(A)$ & negative & positive & negative & negative & negative & negative \\
\hline erm(B) & negative & negative & negative & negative & negative & negative \\
\hline erm(C) & negative & negative & negative & negative & positive & negative \\
\hline
\end{tabular}




\section{Supplementary Information}

Karkaba et al.

New Zealand Veterinary Journal

\begin{tabular}{|c|c|c|c|c|c|c|}
\hline $\ln u(A)$ & negative & negative & negative & negative & negative & negative \\
\hline $\mathrm{msr}(\mathrm{A})$ & negative & negative & negative & negative & negative & negative \\
\hline $\operatorname{mef}(\mathrm{A})$ & negative & negative & negative & negative & negative & negative \\
\hline $\mathrm{mph}(\mathrm{C})$ & negative & negative & negative & negative & negative & negative \\
\hline $\operatorname{vat}(\mathrm{A})$ & negative & negative & negative & negative & negative & negative \\
\hline $\operatorname{vat}(\mathrm{B})$ & negative & negative & negative & negative & negative & negative \\
\hline $\operatorname{vga}(A)$ & negative & negative & negative & negative & negative & negative \\
\hline $\operatorname{vga(A)~(BM~3327)~}$ & negative & negative & negative & negative & negative & negative \\
\hline $\operatorname{vg} B(A)$ & negative & negative & negative & negative & negative & negative \\
\hline \multicolumn{7}{|c|}{ RESISTANCE GENOTYPE : AMINOGLYCOSIDES } \\
\hline Target Gene & Result & Result & Result & Result & Result & Result \\
\hline aacA-aphD & negative & negative & negative & negative & positive & negative \\
\hline aadD & negative & negative & negative & negative & negative & negative \\
\hline aphA3 & negative & negative & negative & negative & negative & negative \\
\hline \multicolumn{7}{|c|}{ RESISTANCE GENOTYPE : MISCELLANEOUS GENES } \\
\hline Target Gene & Result & Result & Result & Result & Result & Result \\
\hline sat & negative & negative & negative & negative & negative & negative \\
\hline dfrS1 & negative & negative & negative & negative & positive & negative \\
\hline fusB & negative & negative & negative & negative & negative & negative \\
\hline Isolate & 1 & $2 \& 3$ & 4 & $5 \& 5.1$ & 6 & a \\
\hline fusC & negative & negative & negative & negative & negative & negative \\
\hline mupA & negative & negative & negative & negative & negative & negative \\
\hline tet $(\mathrm{K})$ & negative & negative & negative & negative & negative & negative \\
\hline tet(M) & negative & negative & negative & negative & positive & negative \\
\hline cat (total) & negative & negative & negative & negative & negative & negative \\
\hline cat (pC221) & negative & negative & negative & negative & negative & negative \\
\hline cat (pc223) & negative & negative & negative & negative & negative & negative \\
\hline cat (pMC524) & negative & negative & negative & negative & negative & negative \\
\hline cat (pSBK203R) & negative & negative & negative & negative & negative & negative \\
\hline $\mathrm{cfr}$ & negative & negative & negative & negative & negative & negative \\
\hline fexA & negative & negative & negative & negative & negative & negative \\
\hline fos $\mathrm{B}$ & positive & positive & positive & negative & positive & negative \\
\hline fosB (plasmid) & negative & negative & negative & negative & negative & negative \\
\hline \multicolumn{7}{|c|}{ RESISTANCE GENOTYPE : EFFLUX SYSTEMS } \\
\hline Target Gene & Result & Result & Result & Result & Result & Result \\
\hline qacA & negative & negative & negative & negative & negative & positive \\
\hline qacC (total) & negative & negative & negative & negative & negative & negative \\
\hline qacC (consensus) & negative & negative & negative & negative & negative & negative \\
\hline qacc (equine) & negative & negative & negative & negative & negative & negative \\
\hline qacC (SA5) & negative & negative & negative & negative & negative & negative \\
\hline qacC (Ssap) & negative & negative & negative & negative & negative & negative \\
\hline qacC (ST94) & negative & negative & negative & negative & negative & negative \\
\hline sdrM & positive & positive & positive & negative & positive & positive \\
\hline \multicolumn{7}{|c|}{ RESISTANCE GENOTYPE : GLYCOPEPTIDES } \\
\hline Target Gene & Result & Result & Result & Result & Result & Result \\
\hline $\operatorname{van} A$ & negative & negative & negative & negative & negative & negative \\
\hline $\operatorname{vanB}$ & negative & negative & negative & negative & negative & negative \\
\hline $\operatorname{vanZ}$ & negative & negative & negative & negative & negative & negative \\
\hline \multicolumn{7}{|l|}{ VIRULENCE: TOXIC SHOCK TOXIN } \\
\hline Target Gene & Result & Result & Result & Result & Result & Result \\
\hline tst1 (consensus) & negative & negative & negative & negative & negative & negative \\
\hline tst1 ("human" allele) & negative & negative & negative & negative & negative & negative \\
\hline tst1 ("bovine" allele, from RF122) & negative & negative & negative & negative & negative & negative \\
\hline \multicolumn{7}{|l|}{ VIRULENCE: ENTEROTOXINS } \\
\hline Target Gene & Result & Result & Result & Result & Result & Result \\
\hline sea & negative & negative & negative & negative & negative & negative \\
\hline
\end{tabular}




\section{Supplementary Information}

Karkaba et al.

New Zealand Veterinary Journal

\begin{tabular}{|c|c|c|c|c|c|c|}
\hline sea (320E) & negative & negative & negative & negative & negative & negative \\
\hline sea (N315) & positive & negative & negative & negative & negative & negative \\
\hline seb & negative & negative & negative & negative & positive & negative \\
\hline sec & positive & negative & negative & positive & negative & negative \\
\hline sed & negative & positive & negative & negative & negative & negative \\
\hline see & negative & negative & negative & negative & negative & negative \\
\hline seg & positive & positive & positive & positive & negative & negative \\
\hline seh & negative & negative & negative & negative & negative & positive \\
\hline sei & positive & positive & positive & positive & negative & negative \\
\hline sej & negative & positive & negative & negative & negative & negative \\
\hline sek & negative & negative & negative & negative & positive & negative \\
\hline sel & positive & negative & negative & positive & negative & negative \\
\hline selm & positive & positive & positive & positive & negative & negative \\
\hline seln (consensus) & positive & positive & positive & positive & negative & negative \\
\hline seln (other than RF122) & positive & positive & positive & positive & negative & negative \\
\hline selo & positive & positive & positive & positive & negative & negative \\
\hline egc & positive & positive & positive & positive & negative & negative \\
\hline seq & negative & negative & negative & negative & positive & negative \\
\hline ser & negative & positive & negative & negative & negative & negative \\
\hline selu & positive & positive & positive & positive & negative & negative \\
\hline Isolate & 1 & $2 \& 3$ & 4 & $5 \& 5.1$ & 6 & $\mathrm{a}$ \\
\hline ORF CM14_probe1 & negative & negative & negative & negative & negative & negative \\
\hline ORF CM14_probe2 & negative & negative & negative & negative & negative & negative \\
\hline \multicolumn{7}{|c|}{ VIRULENCE: HLG AND LEUKOCIDINS } \\
\hline Target Gene & Result & Result & Result & Result & Result & Result \\
\hline lukF & positive & positive & positive & positive & positive & positive \\
\hline lukS & positive & positive & positive & ambiguous & positive & positive \\
\hline lukS (ST22+ST45) & positive & positive & positive & positive & ambiguous & positive \\
\hline hlgA & positive & positive & positive & positive & positive & positive \\
\hline lukF-PV & negative & negative & positive & negative & negative & negative \\
\hline lukS-PV & negative & negative & positive & negative & negative & negative \\
\hline lukF-PV (P83) & negative & negative & negative & negative & negative & positive \\
\hline lukM & negative & negative & negative & negative & negative & positive \\
\hline lukD & positive & positive & negative & negative & positive & positive \\
\hline lukE & positive & positive & negative & negative & positive & positive \\
\hline lukX & positive & positive & positive & positive & positive & positive \\
\hline lukY & positive & positive & negative & positive & positive & positive \\
\hline lukY (ST30+ST45) & negative & negative & positive & negative & negative & negative \\
\hline \multicolumn{7}{|c|}{ VIRULENCE: HAEMOLYSINS } \\
\hline Target Gene & Result & Result & Result & Result & Result & Result \\
\hline $\mathrm{hl}$ & positive & positive & positive & positive & positive & positive \\
\hline hla & positive & positive & positive & positive & positive & positive \\
\hline hllII (consensus) & positive & positive & positive & negative & positive & positive \\
\hline hIIII (other than RF122) & positive & positive & positive & negative & positive & positive \\
\hline hlb_probe 1 & positive & positive & positive & positive & positive & positive \\
\hline hlb_probe 2 & positive & positive & positive & positive & positive & positive \\
\hline hlb_probe 3 & ambiguous & positive & ambiguous & positive & positive & positive \\
\hline un-disrupted hlb & negative & negative & negative & negative & positive & positive \\
\hline \multicolumn{7}{|c|}{ VIRULENCE: HLB-CONVERTING PHAGES } \\
\hline Target Gene & Result & Result & Result & Result & Result & Result \\
\hline sak & positive & positive & positive & positive & negative & negative \\
\hline chp & positive & positive & positive & positive & negative & negative \\
\hline scn & positive & positive & positive & positive & negative & negative \\
\hline \multicolumn{7}{|c|}{ VIRULENCE: EXFOLIATIVE TOXINS } \\
\hline Target Gene & Result & Result & Result & Result & Result & Result \\
\hline etA & negative & negative & negative & negative & negative & negative \\
\hline etB & negative & negative & negative & negative & negative & negative \\
\hline etD & negative & negative & negative & negative & negative & negative \\
\hline
\end{tabular}




\section{Supplementary Information}

Karkaba et al.

New Zealand Veterinary Journal

VIRULENCE: EPIDERMAL CELL DIFFERENTION INHIBITORS

\begin{tabular}{l|l|l|l|l|l|l|l}
\hline Target Gene & Result & Result & Result & Result & Result & Result \\
\hline edinA & negative & negative & negative & negative & negative & negative \\
\hline edinB & negative & negative & negative & negative & negative & negative \\
\hline edinC & negative & negative & negative & negative & negative & negative \\
\hline VIRULENCE: ACME LOCUS & \multicolumn{5}{|l}{} \\
\hline Target Gene & Result & Result & Result & Result & \multicolumn{2}{l}{ Result } & Result \\
\hline ACME cluster & negative & negative & negative & negative & negative & negative \\
\hline arCA-SCC & negative & negative & negative & negative & negative & negative \\
\hline arCB-SCC & negative & negative & negative & negative & negative & negative \\
\hline arCC-SCC & negative & negative & negative & negative & negative & negative \\
\hline arCD-SCC & negative & negative & negative & negative & negative & negative \\
\hline
\end{tabular}

VIRULENCE: PROTEASES

\begin{tabular}{l|l|l|l|l|l|l}
\hline Target Gene & Result & Result & Result & Result & Result & Result \\
\hline aur (consensus) & positive & positive & positive & positive & positive & positive \\
\hline aur (other than MRSA252) & positive & positive & negative & negative & positive & positive \\
\hline aur (MRSA252) & negative & negative & positive & positive & negative & negative \\
\hline spIA & positive & positive & negative & negative & positive & positive \\
\hline splB & positive & positive & negative & negative & positive & positive \\
\hline splE & negative & negative & positive & negative & positive & positive \\
\hline Isolate & 1 & $2 \& 3$ & 4 & $5 \& 5.1$ & 6 & a \\
\hline & & & & & & \\
\hline sspA & positive & positive & positive & positive & positive & positive \\
\hline sspB & positive & positive & positive & positive & positive & positive \\
\hline sspP (consensus) & positive & positive & positive & positive & positive & positive \\
\hline SspP (other than ST93) & positive & positive & positive & positive & positive & positive
\end{tabular}

\begin{tabular}{|c|c|c|c|c|c|c|}
\hline Target Gene & Result & Result & Result & Result & Recult & Recult \\
\hline setC/selx & positive & positive & negative & positive & positive & positive \\
\hline ssl01/set6_probe1_11 & positive & positive & negative & negative & positive & negative \\
\hline ssl01/set6_probe2_11 & negative & negative & positive & positive & negative & positive \\
\hline ssl01/set6_probe1_12 & positive & positive & positive & positive & negative & negative \\
\hline ssl01/set6_probe2_12 & negative & negative & negative & negative & negative & positive \\
\hline ssl01/set6_probe4_11 & positive & positive & positive & negative & positive & negative \\
\hline ssl01/set6_probeRF122 & negative & negative & negative & negative & negative & negative \\
\hline ssl01/set6 (COL) & negative & negative & negative & negative & positive & negative \\
\hline ssl01/set6 (Mu50+N315) & positive & positive & negative & negative & ambiguous & negative \\
\hline ssl01/set6 (MW2+MSSA476) & negative & negative & negative & negative & negative & positive \\
\hline ssl01/set6 (MRSA252) & negative & negative & positive & positive & negative & negative \\
\hline ssl01/set6 (RF122) & negative & negative & negative & negative & negative & negative \\
\hline ssl01/set6 (other alleles) & negative & negative & negative & negative & negative & negative \\
\hline ssl02/set7 & positive & positive & negative & ambiguous & positive & positive \\
\hline ssl02/set7 (MRSA252) & negative & ambiguous & positive & positive & negative & negative \\
\hline ssl03/set8_probe 1 & positive & positive & negative & negative & positive & positive \\
\hline ssl03/set8_probe 2 & positive & positive & negative & negative & positive & positive \\
\hline ssl03/set8 (MRSA252, SAR0424) & negative & negative & positive & negative & negative & negative \\
\hline ssl04/set9 & negative & positive & negative & negative & positive & positive \\
\hline ssl04/set9 (MRSA252, SAR0425) & negative & negative & positive & positive & negative & negative \\
\hline ssl05/set3_probe 1 & positive & positive & negative & positive & positive & positive \\
\hline ssl05/set3 (RF122, probe-611) & ambiguous & ambiguous & negative & negative & negative & ambiguous \\
\hline ssl05/set3_probe 2 (612) & positive & positive & negative & negative & positive & positive \\
\hline ssl05/set3 (MRSA252) & negative & negative & positive & positive & negative & negative \\
\hline ssl06/set21 & negative & negative & negative & negative & positive & positive \\
\hline ssl06 (NCTC8325+MW2) & negative & negative & negative & negative & positive & positive \\
\hline ssl07/set1 & positive & positive & negative & ambiguous & positive & positive \\
\hline ssl07/set1 (MRSA252) & ambiguous & ambiguous & positive & ambiguous & ambiguous & ambiguous \\
\hline ssl07/set1 (AF188836) & negative & negative & negative & positive & negative & negative \\
\hline ssl08/set12_probe 1 & positive & positive & negative & negative & positive & positive \\
\hline
\end{tabular}




\section{Supplementary Information}

\begin{tabular}{|c|c|c|c|c|c|c|}
\hline ssl08/set12_probe 2 & positive & positive & negative & negative & positive & positive \\
\hline ssl09/set5_probe 1 & positive & positive & negative & positive & positive & positive \\
\hline ssl09/set5_probe 2 & positive & positive & negative & positive & positive & positive \\
\hline ssl09/set5 (MRSA252) & negative & negative & positive & negative & negative & negative \\
\hline ssl10/set4 & positive & positive & ambiguous & positive & positive & positive \\
\hline ssl10 (RF122) & negative & negative & negative & negative & negative & negative \\
\hline ssl10/set4 (MRSA252) & ambiguous & ambiguous & positive & negative & ambiguous & ambiguous \\
\hline ssl11/set2 (COL) & negative & negative & negative & negative & positive & negative \\
\hline ssl11+set2(Mu50+N315) & positive & positive & negative & negative & negative & negative \\
\hline ssl11+set2(MW2+MSSA476) & negative & negative & negative & negative & negative & positive \\
\hline ssl11/set2 (MRSA252) & negative & negative & positive & negative & negative & negative \\
\hline setB3 & positive & positive & negative & negative & positive & positive \\
\hline setB3 (MRSA252) & negative & negative & positive & negative & negative & negative \\
\hline setB2 & positive & positive & negative & negative & positive & positive \\
\hline setB2 (MRSA252) & negative & negative & positive & negative & negative & negative \\
\hline setB1 & positive & positive & positive & negative & positive & positive \\
\hline \multicolumn{7}{|c|}{ CAPSULE- AND BIOFILM-ASSOCIATED GENES } \\
\hline Target Gene & Result & Result & Result & Result & Result & Result \\
\hline cap 1 (total) & negative & negative & negative & negative & negative & negative \\
\hline Isolate & 1 & $2 \& 3$ & 4 & $5 \& 5.1$ & 6 & a \\
\hline Isolate & 1 & $2 \& 3$ & 4 & $5 \& 5.1$ & 6 & $\mathrm{a}$ \\
\hline capH1 & negative & negative & negative & negative & negative & negative \\
\hline capJ1 & negative & negative & negative & negative & negative & negative \\
\hline capK1 & negative & negative & negative & negative & negative & negative \\
\hline cap 5 (total) & positive & positive & negative & positive & positive & negative \\
\hline capH5 & positive & positive & negative & positive & positive & negative \\
\hline capJ5 & positive & positive & negative & positive & positive & negative \\
\hline capK5 & positive & positive & negative & positive & positive & negative \\
\hline cap 8 (total) & negative & negative & positive & negative & negative & positive \\
\hline capH8 & negative & negative & positive & negative & negative & positive \\
\hline capl8 & negative & negative & positive & negative & negative & positive \\
\hline capJ8 & negative & negative & positive & negative & negative & positive \\
\hline capK8 & negative & negative & positive & negative & negative & positive \\
\hline icaA & positive & positive & positive & positive & positive & positive \\
\hline icaC & positive & positive & positive & positive & positive & positive \\
\hline icaD & positive & positive & positive & positive & positive & positive \\
\hline bap & negative & negative & negative & negative & negative & negative \\
\hline \multicolumn{7}{|c|}{$\begin{array}{l}\text { ADHAESION FACTORS I GENES ENCODING MICROBIAL SURFACE COMPONENTS RECOGNIZING ADHESIVE MATRIX MOLECULES } \\
\text { (MSCRAMM GENES) }\end{array}$} \\
\hline Target Gene & Result & Result & Result & Result & Result & Result \\
\hline bbp (total) & positive & positive & positive & positive & positive & positive \\
\hline bbp (consensus) & positive & positive & positive & positive & positive & positive \\
\hline $\mathrm{bbp}(\mathrm{COL}+\mathrm{MW} 2)$ & negative & negative & negative & negative & positive & positive \\
\hline bbp (MRSA252) & negative & negative & positive & negative & negative & negative \\
\hline bbp (Mu50) & positive & positive & negative & negative & negative & negative \\
\hline bbp (RF122) & negative & negative & negative & negative & negative & negative \\
\hline bbp (ST45) & negative & negative & negative & negative & negative & negative \\
\hline clfA (total) & positive & positive & positive & positive & positive & positive \\
\hline clfA (consensus) & positive & positive & positive & positive & positive & positive \\
\hline clfA (COL+RF122) & negative & negative & ambiguous & negative & positive & negative \\
\hline clfA (MRSA252) & negative & negative & positive & negative & ambiguous & negative \\
\hline clfA (Mu50+MW2) & positive & positive & negative & positive & ambiguous & positive \\
\hline clfB (total) & positive & positive & positive & positive & positive & positive \\
\hline clfB (consensus) & positive & positive & positive & positive & positive & positive \\
\hline clfB (COL+Mu50) & positive & positive & negative & negative & positive & negative \\
\hline clfB (MW2) & negative & negative & negative & negative & negative & positive \\
\hline
\end{tabular}




\section{Supplementary Information}

\begin{tabular}{|c|c|c|c|c|c|c|}
\hline clfB (RF122) & negative & negative & negative & negative & negative & ambiguous \\
\hline cna & negative & negative & positive & positive & negative & positive \\
\hline ebh (consensus) & positive & positive & positive & negative & positive & positive \\
\hline ebpS (total) & positive & positive & positive & positive & positive & positive \\
\hline ebpS_probe 612 & positive & positive & positive & positive & positive & positive \\
\hline ebpS_probe 614 & positive & positive & positive & positive & positive & positive \\
\hline ebpS (01-1111) & negative & negative & negative & negative & negative & negative \\
\hline ebpS (COL) & negative & negative & negative & positive & positive & negative \\
\hline eno & positive & positive & positive & positive & positive & positive \\
\hline $\mathrm{fib}$ & positive & positive & negative & negative & positive & positive \\
\hline fib (MRSA252) & negative & negative & positive & positive & negative & negative \\
\hline fnbA (total) & positive & positive & positive & positive & positive & positive \\
\hline fnbA (consensus) & positive & positive & positive & positive & positive & positive \\
\hline fnbA (COL) & negative & negative & negative & negative & positive & negative \\
\hline fnbA (MRSA252) & negative & negative & positive & negative & negative & negative \\
\hline fnbA (Mu50+MW2) & positive & positive & negative & positive & negative & positive \\
\hline fnbA (RF122) & negative & negative & negative & negative & negative & negative \\
\hline fnbB (total) & positive & positive & positive & negative & positive & positive \\
\hline fnbB (COL) & negative & negative & negative & negative & positive & negative \\
\hline fnbB (COL+Mu50+MW2) & positive & ambiguous & ambiguous & negative & ambiguous & ambiguous \\
\hline fnbB (Mu50) & ambiguous & positive & positive & negative & negative & negative \\
\hline fnbB (MW2) & negative & negative & negative & negative & negative & positive \\
\hline Isolate & 1 & $2 \& 3$ & 4 & $5 \& 5.1$ & 6 & $a$ \\
\hline fnbB (ST15) & negative & negative & negative & negative & negative & negative \\
\hline fnbB (ST45-2) & negative & negative & negative & negative & negative & negative \\
\hline map (total) & positive & positive & positive & positive & positive & positive \\
\hline map (COL) & ambiguous & positive & negative & negative & positive & negative \\
\hline map (MRSA252) & negative & negative & positive & negative & negative & negative \\
\hline map (Mu50+MW2) & positive & positive & negative & positive & negative & positive \\
\hline sasG (total) & positive & positive & negative & positive & positive & positive \\
\hline sasG (COL+Mu50) & positive & positive & negative & negative & positive & negative \\
\hline sasG (MW2) & negative & negative & negative & positive & negative & positive \\
\hline sasG (other than MRSA252+RF122) & positive & positive & negative & positive & positive & positive \\
\hline sdrC (total) & positive & positive & positive & positive & positive & positive \\
\hline sdrC (consensus) & positive & positive & positive & positive & positive & positive \\
\hline sdrC (B1) & negative & negative & negative & negative & negative & negative \\
\hline $\operatorname{sdrC}(\mathrm{COL})$ & negative & negative & negative & negative & positive & negative \\
\hline sdrC (Mu50) & positive & positive & negative & positive & negative & negative \\
\hline sdrC (MW2+MRSA252+RF122) & negative & negative & negative & negative & negative & positive \\
\hline sdrC (other than MRSA252+RF122) & positive & positive & negative & positive & positive & positive \\
\hline sdrD (total) & positive & positive & positive & positive & positive & positive \\
\hline sdrD (consensus) & positive & positive & positive & positive & positive & positive \\
\hline sdrD (COL+MW2) & negative & negative & negative & negative & positive & positive \\
\hline sdrD (Mu50) & positive & positive & negative & negative & negative & negative \\
\hline sdrD (other) & negative & negative & positive & positive & negative & negative \\
\hline vwb (total) & positive & positive & positive & positive & positive & positive \\
\hline vwb (consensus) & positive & positive & positive & positive & positive & positive \\
\hline vwb (COL+MW2) & negative & negative & negative & negative & positive & positive \\
\hline vwb (MRSA252) & negative & negative & positive & negative & negative & negative \\
\hline vwb (Mu50) & positive & positive & negative & negative & negative & negative \\
\hline vwb (RF122) & negative & negative & negative & positive & negative & negative \\
\hline \multicolumn{7}{|l|}{ IMMUNODOMINANT ANTIGEN B } \\
\hline Target Gene & Result & Result & Result & Result & Result & Result \\
\hline isaB & positive & positive & negative & negative & positive & positive \\
\hline isaB (MRSA252) & ambiguous & ambiguous & positive & positive & ambiguous & ambiguous \\
\hline \multicolumn{7}{|c|}{ DEFENSIN RESISTANCE GENOTYPE } \\
\hline Target Gene & Result & Result & Result & Result & Result & Result \\
\hline
\end{tabular}




\section{Supplementary Information}

\begin{tabular}{|c|c|c|c|c|c|c|}
\hline mprF (COL+MW2) & ambiguous & ambiguous & ambiguous & ambiguous & positive & positive \\
\hline mprF (Mu50+MRSA252) & positive & positive & positive & positive & ambiguous & ambiguous \\
\hline \multicolumn{7}{|l|}{ TRANSFERRIN BINDING PROTEIN } \\
\hline Target Gene & Result & Result & Result & Result & Result & Result \\
\hline isdA (consensus) & positive & positive & positive & positive & positive & positive \\
\hline isdA (MRSA252) & negative & negative & positive & negative & negative & negative \\
\hline isdA (other than MRSA252) & positive & positive & negative & positive & positive & positive \\
\hline \multicolumn{7}{|l|}{ PUTATIVE TRANSPORTER } \\
\hline Target Gene & Result & Result & Result & Result & Result & Result \\
\hline ImrP (other than RF122)_probe1 & positive & positive & positive & positive & positive & positive \\
\hline ImrP (other than RF122)_probe2 & positive & positive & positive & positive & positive & positive \\
\hline ImrP (RF122)_probe1 & negative & negative & negative & negative & negative & negative \\
\hline ImrP (RF122)_probe2 & negative & negative & negative & negative & negative & negative \\
\hline \multicolumn{7}{|c|}{ TYPE I RESTRICTION-MODIFICATION SYSTEM, SINGLE SEQUENCE SPECIFICITY PROTEIN } \\
\hline Target Gene & Result & Result & Result & Result & Result & Result \\
\hline hsdS1 (RF122) & negative & negative & negative & negative & negative & negative \\
\hline $\begin{array}{l}\text { hsdS2 } \\
\text { (Mu50+N315+COL+USA300+NCTC } \\
8325)\end{array}$ & positive & positive & negative & positive & positive & negative \\
\hline hsdS2 (MW2+MSSA476) & ambiguous & negative & negative & negative & negative & positive \\
\hline hsdS2 (RF122) & negative & negative & negative & negative & negative & negative \\
\hline hsdS2 (MRSA252) & negative & negative & positive & negative & negative & negative \\
\hline $\begin{array}{l}\text { hsdS3 (all other than RF122+ } \\
\text { MRSA252) }\end{array}$ & positive & positive & negative & negative & negative & positive \\
\hline Isolate & 1 & $2 \& 3$ & 4 & $5 \& 5.1$ & 6 & a \\
\hline $\begin{array}{l}\text { hsdS3 } \\
\text { (COL+USA300+NCTC8325+MW2+ } \\
\text { MSSA476+RF122) }\end{array}$ & negative & negative & negative & negative & negative & positive \\
\hline hsdS3 (Mu50+N315) & positive & positive & negative & negative & negative & negative \\
\hline hsdS3 (CC51+ MRSA252) & negative & negative & positive & negative & negative & negative \\
\hline hsdS3 (MRSA252) & negative & negative & positive & negative & negative & negative \\
\hline hsdSx (CC25) & positive & positive & positive & positive & ambiguous & positive \\
\hline hsdSx (CC15) & negative & negative & negative & negative & negative & negative \\
\hline hsdSx (etd) & negative & negative & negative & negative & negative & negative \\
\hline \multicolumn{7}{|l|}{ MISCELLANEOUS GENES } \\
\hline Target Gene & Result & Result & Result & Result & Result & Result \\
\hline Q2FXC0 & negative & negative & negative & negative & positive & positive \\
\hline Q2YUB3 & negative & negative & negative & negative & negative & positive \\
\hline Q7A4X2 & positive & positive & positive & positive & negative & negative \\
\hline \multicolumn{7}{|l|}{ HYALURONATE LYASE } \\
\hline Target Gene & Result & Result & Result & Result & Result & Result \\
\hline hysA1 (MRSA252) & negative & negative & positive & negative & negative & negative \\
\hline $\begin{array}{l}\text { hysA1 (MRSA252+RF122) and/or } \\
\text { hysA2 (consensus) }\end{array}$ & positive & positive & positive & positive & positive & positive \\
\hline $\begin{array}{l}\text { hysA1 (MRSA252+RF122) and/or } \\
\text { hysA2 (COL+USA300) }\end{array}$ & negative & negative & positive & negative & positive & negative \\
\hline hysA2 (all other than MRSA252) & positive & positive & negative & negative & positive & positive \\
\hline hysA2 (COL+USA300+NCTC8325) & negative & negative & positive & negative & positive & negative \\
\hline $\begin{array}{l}\text { hysA2 (all other than } \\
\text { COL+USA300+NCTC8325)_probe1 }\end{array}$ & positive & positive & positive & positive & negative & positive \\
\hline $\begin{array}{l}\text { hysA2 (all other than } \\
\text { COL+USA300+NCTC8325)_probe2 }\end{array}$ & positive & positive & positive & positive & negative & positive \\
\hline hysA2 (MRSA252) & negative & negative & positive & negative & negative & negative \\
\hline
\end{tabular}

